\title{
Analyse de la Structure Morphologique des Cernes de Croissance et Détermination Des Âges de Deux Espèces Sahéliennes : Acacia Tortilis (Forsk.) Hayne Ssp. Raddiana (Savi) Brenan, Acacia Senegal (L.) Willd
}

\author{
Daouda Ndiaye, \\ Moustapha Bassimbé Sagn,
}

Département de Biologie Végétale, Faculté des Sciences et Techniques, Université Cheikh Anta Diop, Dakar- Sénégal,

Aly Diallo,

Université Assane Seck de Ziguinchor

Sékouna Diat,

Département de Biologie Végétale, Faculté des Sciences et Techniques,

Université Cheikh Anta Diop, Dakar- Sénégal,

Jean Luc Peiry, PhD

UMI 3189/CNRS, Université de Clermont Ferrand-Geolab-UMR

Aliou Guissé, PhD

Département de Biologie Végétale, Faculté des Sciences et Techniques,

Université Cheikh Anta Diop, Dakar- Sénégal

\section{Résumé}

La connaissance de la dendrochronologie des espèces sahéliennes est très limitée. Néanmoins, elle peut constituer une voie pour mieux améliorer la connaissance de la dynamique de croissances des espèces en zone sèche africaine. Cette étude vise à mieux connaitre la structure anatomique des cernes de croissances d'espèces sahéliennes comme moyen de datation et d'estimation de l'âge des arbres et arbustes. Elle a été réalisée dans la zone du Ferlo situé au Nord du Sénégal sur 14 et 12 rondelles de bois de Acacia tortilis (Forsk.) Hayne ssp. raddiana (Savi) Brenan, et de Acacia senegal (L.)Willd. Des méthodes d'identification et de datation des cernes ont été utilisées en vue d'étudier leur structure morpho-anatomiques des cernes et de déterminer l'âge des individus. Les cernes ont été identifiés et des photos à très haute résolution (1200 dpi) ont été prises à l'aide d'une loupe binoculaire Leica (M80) équipée d'une caméra digitale. Les photos prises ont été assemblées avec le logiciel 
Adobe Photoshop. Les résultats ont montré que les deux espèces présentent des cernes de croissances distinctes marquées par des cellules de parenchymes. Des corrélations statistiquement significatives comprises entre 0,33 à 0,82 ont ainsi été calculées entre les rayons d'un même individu. Les âges estimés chez les Acacia raddiana sont compris entre 10 à 27 ans alors que les Acacia senegal paraissent plus âgés entre 13 à 35 ans. La lecture des cernes plus ou moins difficile chez les Acacias est liée à la structure anatomique du bois dont les limites entre les cernes sont souvent cachées par des groupes de parenchymes. Cette étude contribue à mettre en place des stratégies et des méthodes permettant de maitriser la dynamique de croissance de ces espèces afin de les valoriser.

Mots-clés : A. Raddiana, A. Senegal, Datation, Estimation, Cernes, Âges 


\title{
Analysis of the Morphological Structure of the Growth Rings and Determination of the Ages of Two Sahelian Species: Acacia Tortilis (Forsk.) Hayne Ssp. Raddiana (Savi) Brenan, and Acacia Senegal (L.) Willd
}

\author{
Daouda Ndiaye, \\ Moustapha Bassimbé Sagn,
}

Département de Biologie Végétale, Faculté des Sciences et Techniques, Université Cheikh Anta Diop, Dakar- Sénégal,

Aly Diallo,

Université Assane Seck de Ziguinchor

Sekouna Diat,

Département de Biologie Végétale, Faculté des Sciences et Techniques,

Université Cheikh Anta Diop, Dakar- Sénégal,

Jean Luc Peiry, PhD

UMI 3189/CNRS, Université de Clermont Ferrand-Geolab-UMR 6042 Aliou Guisse, PhD

Département de Biologie Végétale, Faculté des Sciences et Techniques,

Université Cheikh Anta Diop, Dakar- Sénégal

\begin{abstract}
Knowledge of the dendrochonology of Sahelian species is very limited. However, dendrochronology may be a way of furthering the knowledge of the growth dynamics the African dry area species. The purpose of the study was to better understand the anatomical structure of the tress rings of the Sahelian species in order to determine the age and estimation of trees and shrubs. It was conducted out in the Ferlo, an area located in the north of Senegal, and focused on 14 and 12 wood slices of Acacia tortilis (Forsk.) Hayne ssp. raddiana (Savi) Brenan and from Acacia senegal (L.) Willd respectively. Identification and dating methods were used to study the morpho-anatomical structure of the ring and determine the age of individuals. The rings were identified and very high-resolution (1200 dpi) pictures taken with a Leica (M80) binocular magnifier equipped with a digital camera. Then the pictures were assembled with the Adobe Photoshop software.The results showed that both species had distinct growth rings with parenchymal cells.
\end{abstract}


Significant correlations between 0.33 and 0.82 was then calculated between the radii of the same individual. Estimated ages of the Acacia raddiana ranged from 10 to 27 years, whereas the Acacia senegal seemed older white 13 to 35 years of age. The difficulty to read the rings of the Acacias is related to the anatomical structure of the wood because boundaries between dark circles are often hidden by parenchyma clusters. The study helps put in place strategies and methods which enable to control the growth dynamics of the species in order to valorize them.

Keywords: A. Raddiana, A. Senegal, Datation, Estimation, Tree Rings, Age

\section{Introduction}

Depuis plusieurs décennies, les forêts tropicales et surtout les écosystèmes sahéliens sont en perpétuelles déperditions (Henry et al., 2010). Au cours des années 1970, un large mouvement ayant pour vocation la protection de la nature, en général, a vu le jour dans de nombreux pays. On peut citer l'exemple de l'UNESCO dans le cadre du programme sur l'homme et la biosphère (ou programme MAB pour Man and Biosphere) et celui de la Convention concernant le Patrimoine Mondial. Dans ce contexte, la protection des espèces consiste avant tout à entretenir, conserver et éventuellement rétablir les biotopes naturels. Dans les zones sèches d'Afrique, certaines espèces sont caractéristiques des écosystèmes sahéliens; il s'agit principalement des espèces adaptées à l'aridité comme Acacia tortilis (forsk.) Hayne ssp. raddiana (Savi) Brenan et Acacia senegal (L.)Willd. Elles assurent avec d'autres, des services très importants pour la population locale. Elles sont utilisées comme fourrage, leur bois sert de charbon de chauffage (Guérin et al.,1991, Ngom et al., 2014). Ces essences constituent des espèces à usages multiples (Sarr et al., 2014) et sont donc très prisées. Ainsi, les Acacia sont victimes d'une forte exploitation surtout pour leur gomme arabique (Dione, 1996 ; Séne, 1998 ; Diallo et al., 2011). Face à cette forte pression anthropique, la valorisation de ces essences précieuses est essentielle, car elle constitue la meilleure garantie pour le maintien de la capacité de charge des populations pour une exploitation durable, voire un maintien à long terme des individus (Diop, 2007). En effet, face à l'insuffisance des stratégies d'assistance à la régénération naturelle et au reboisement spécifique peu développés, la connaissance de la vitesse de croissance et la structure du bois des peuplements ligneux permettraient de mieux gérer la planification de l'exploitation de ces espèces sans risque de compromettre leur pérennité. Ainsi, la dendrochronologie utilisant les fluctuations de la croissance des arbres dans le temps (Cook et Kairiukstis, 1990), est une des méthodes de datation permettant de déterminer la durée de vie d'un arbre et de préciser l'année et la saison de son abattage. Elle offre aussi un moyen de vérifier cette 
hypothèse en milieu naturel, car les données de croissance radiale peuvent être interprétées comme des indicateurs de la croissance et de la productivité de nos écosystèmes sahéliens. Elle sera réalisée grâce à la lecture de cernes, la détermination de l'âge des individus, la description de l'anatomie des cernes de croissance et leur corrélation avec les facteurs environnementaux impliqués dans leur croissance. Depuis les travaux de Coster, 1927 à 1928 ; Worbes, 1989 ; Dezzeo et al., 2003 ; Fichtler et al., 2006 ; Patrut et al., 2007 mettant en évidence les cernes annuels dans des arbres de zones tropicales, plusieurs chercheurs ont tenté de démontrer l'existence de cernes annuels dans les bois d'espèces tropicales, mais peu se sont intéressés aux arbres des zones sèches africaines à l'exception de Mariaux $(1967 ; 1969 ; 1970 ; 1975)$, Worbes (1989), Tarhule et Hughes (2002) et de Mbow (2009). Face à cette connaissance limitée de l'anatomie des cernes de croissance et de la structure du bois de certaines espèces sahéliennes, cette étude vise à montrer qu'endehors des nombreuses thématiques de recherches développées sur ces espèces, un autre axe de recherche pourrait être exploré et intégré ; il s'agit de l'étude de leur potentielle dendrochronologique. C'est dans ce cadre que cette étude a été initiée dans le but de déterminer la structure morphologique des cernes de croissance, mettre en évidence le caractère annuel des cernes de croissance et estimer l'âge des individus de chaque espèce.

\section{Matériel et méthode Sites d'étude}

Les échantillons d'Acacia étudiés ont été collectés dans une zone semidésertique située au nord du Sénégal communément appelé le Ferlo (Figure 1). Avec une superficie de $70000 \mathrm{~km}^{2}$ (Miehe et al., 2010), cette vaste région fait partie de l'immense zone sahélienne. Du point de vue climatique, la pluviométrie annuelle est supérieure à $350 \mathrm{~mm}$ (Sarr, 2009 ; Peiry et Voldoire, 2018). Le diagramme ombrothermique de la région de Linguère qui couvre la zone échantillonnée révèle une saison des pluies concentrée sur trois (03) à quatre (04) mois (août, septembre et octobre) (Figure 1). L'humidité relative de l'air est très faible (moyenne annuelle $35 \%$ ) avec une forte évapotranspiration allant de 1800 à 2200 mm/an (Akpo et al., 1995 ; Sarr, 2009 ; Peiry et Voldoire, 2018).

Le Ferlo abrite une steppe arborée à arbustive dominée par les ligneux tels que Balanites aegyptiaca (L.) Del, Boscia senegalensis (Pers.) Lam. Ex Poir, Acacia senegal (L.), Acacia tortilis (forsk.) Hayne ssp. raddiana (Savi) Brenan, et Calotropis procera (Aiton) W.T.Aiton (Akpo et al., 1995 ; Niang, 2009 ; Miehe et al,. 2010 ; Ndiaye, 2013).

Cette végétation est inféodée à deux types de sols (Leprun, 1971):

- les sols sableux du système dunaire constitués de sols brun-rouge sabloargileux et pauvres en matières organiques ; 
- les sols ferrugineux tropicaux sableux à sablo-argileux rouge, plus ou moins lessivés et pauvres en matières organiques.

C'est dans cet écosystème que cette étude a été réalisée sur deux sites distants de 70 km au Sud de Widou-Thiengoly et au Nord-Est de Linguère (Figure 1). Ces sites ont été choisis en raison de la densité importante des peuplements des Acacia (Ndiaye et al., 2013).

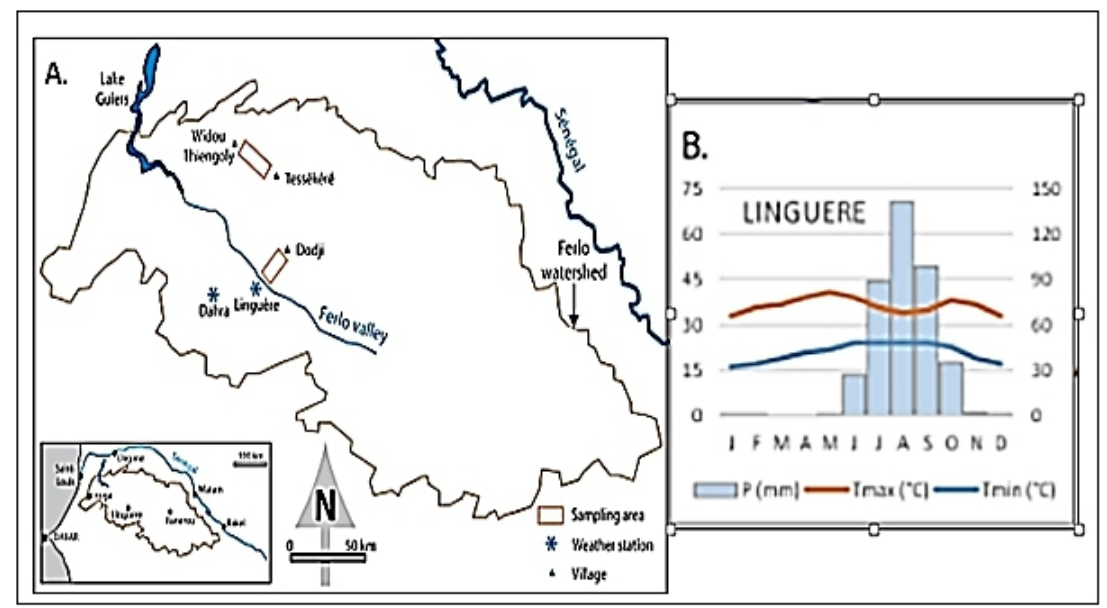

Figure 1. Localisation des zones échantillonnées (A) ; diagramme ombrothermique de la station météorologique de Linguère de 1970 à 2012(B). (Peiry et Voldoire, 2018)

\section{Matériel}

Les populations ciblées par cette étude appartiennent à la même famille (Mimosaceae), au même genre (Acacia) mais à des espèces différentes. Il s'agit de Acacia tortilis (forsk.) Hayne ssp. raddiana (Savi) Brenan, Acacia senegal (L.)

Acacia tortilis (forsk.) Hayne ssp. raddiana (photo 1) est un arbuste ou arbre à tronc légèrement tortueux. Son écorce est généralement grise à brun-noir ou foncé rugueuse, fissurée ou lisse (Diouf, 2003). L'espèce est bien répartie dans la zone Soudano-sahélienne d'Afrique et d'Asie (Vassal, 1993). Elle présente des intérêts (fourrage et domestique) avérés (Chistopher, 1991 ; Dommergues et al., 1999), excellent bois de feu et du charbon de très bonne qualité. Elle est à la base de plusieurs produits de la pharmacopée traditionnelle (Von Maydell, 1990). 
A. senegal (L.) Willd (Photo 2) est également un arbuste ou arbre, avec un tronc plus ou moins asymétrique et l'écorce est généralement de gris-blanc et est rencontrée dans la zone soudano-sahélienne (Afrique) et en Asie (Vassal J., 1996). Elle est utilisée dans l'alimentation humaine et animale (Guinko et al., 1990), la fertilisation des sols en tant que légumineuse pérenne. Son bois est d'usages multiples (bois de service, bois de chauffe, charbon de bois et bois artisanal), (Diallo, 2011). Les arbres et arbustes sont caractérisés par leur capacité à produire de la gomme arabique utilisée dans les industries pharmaceutique, alimentaire, cosmétique et textile (Poupon, 1979; Diallo et al., 2011).

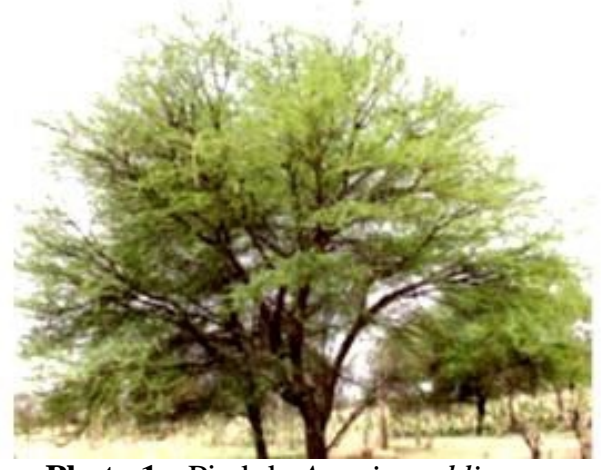

Photo 1 : Pied de Acacia raddiana

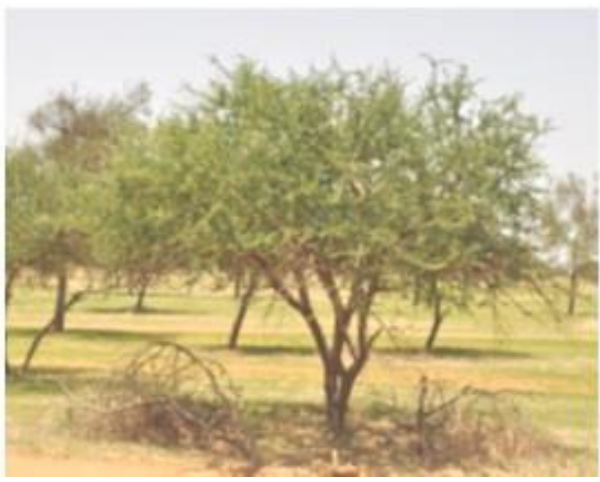

Photo 2 : Pied de Acacia senegal

(Source : Photos prisent lors des travaux de terrain en 2012)

Le prélèvement d'échantillons destinés à des analyses au laboratoire a nécessité le matériel suivant (photos 3 à 6) :

$\checkmark$ une tronçonneuse pour couper les rondelles de bois ;

$\checkmark$ ponceuse orbitale (photo 3) pour poncer afin d'améliorer la visibilité des cernes ;

$\checkmark$ des papiers en verre fin distique (photo 4) pour polir la surface transversale des rondelles de bois (photo 5) ;

$\checkmark$ et enfin une loupe binoculaire Leica (Modèle M80 ; photo 6) pour détection des cernes. 


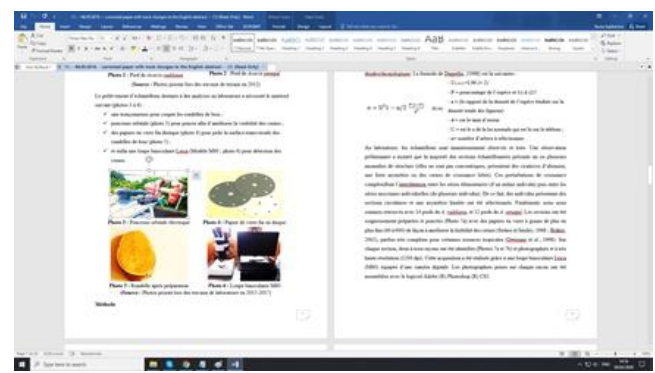

Photo 3 : Ponceuse orbitale électrique

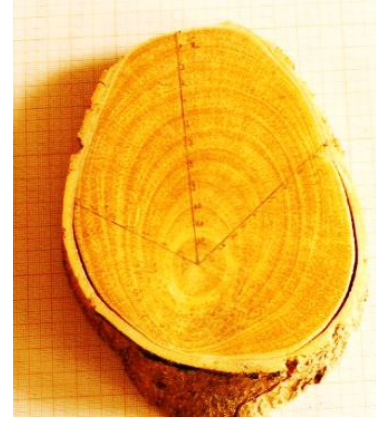

Photo 5 : Rondelle après préparation

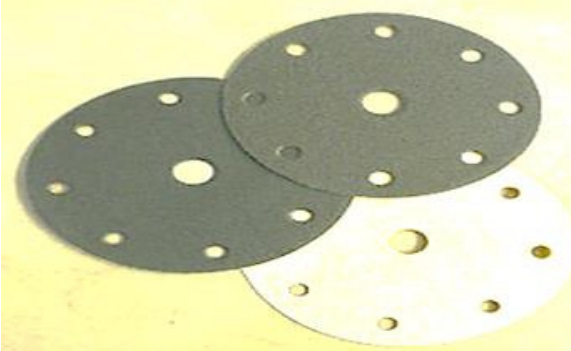

Photo 4 : Papier de verre fin en disque

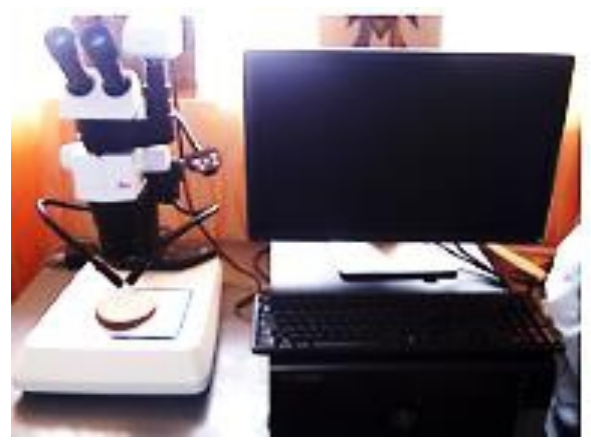

Photo 6 : Loupe binoculaire M80

(Source : Photos prisent lors des travaux de laboratoire en 2015-2017)

\section{Méthode}

\section{Prélèvements et préparation des échantillons}

Des arbres et arbustes ont été coupés lors d'une unique campagne d'abattage réalisée en août 2012. Des sections transversales de 2 à $4 \mathrm{~cm}$ d'épaisseur ont été prélevées sur les troncs de chaque pied à deux niveaux : à $0,30 \mathrm{~m}$ et à $1,30 \mathrm{~m}$ du sol. La taille de l'échantillon a été de trente-huit (38) pieds pour A. raddiana, et soixante-cinq (65) pieds pour A. senegal. Elle a été calculée à partir de la formule de Dagnélie, (1998) pour les besoins de notre étude menés sur l'évaluation de biomasse aérienne de ces espèces. C'est suite à ce travail, que les rondelles de bois provenant de la mesure de biomasse seront utilisées pour une analyse dendrochronologique. La formule de Dagnélie, (1998) est la suivantes :

$$
\begin{array}{ll} 
& -\mathrm{U}_{1-\alpha / 2}=1,96(\approx 2) \\
& -\mathrm{P}=\text { pourcentage de l'espèce et } 1 \leq \mathrm{d} \leq 15 \\
& -\mathrm{a}=(\text { le rapport de la densité de l'espèce } \\
n=\mathrm{U}^{2} 1-\alpha / \quad \text { Avec } & \text { étudiée sur la densité totale des ligneux }) \\
2 \frac{P(1-P)}{d^{2}} & -\mathrm{d}=\text { est le taux d'erreur } \\
& -\mathrm{U}=\text { est le } \mathrm{u} \text { de la loi normale qui est lu } \\
& \text { sur le tableau } ;-\mathrm{n}=\text { nombre d'arbres à } \\
& \text { sélectionner. }
\end{array}
$$


$\mathrm{Au}$ laboratoire, les échantillons sont minutieusement observés et triés. Une observation préliminaire a montré que la majorité des sections échantillonnées présente un ou plusieurs anomalies de structure (elles ne sont pas concentriques, présentent des cicatrices d'abrasion, une forte asymétrie ou des cernes de croissance lobés). Ces perturbations de croissance complexifient l'interdatation entre les séries élémentaires (d'un même individu) puis entre les séries moyennes individuelles (de plusieurs individus). De ce fait, des individus présentant des sections circulaires et une asymétrie limitée ont été sélectionnés. Finalement, nous nous sommes retrouvés avec 14 pieds de $A$. raddiana, et 12 pieds de $A$. senegal. Les sections ont été soigneusement préparées et poncées (Photo $7 \mathrm{a}$ ) avec des papiers en verre à grains de plus en plus fins (60 à 600) de façon à améliorer la lisibilité des cernes (Stokes et Smiley, 1968 ; Bräker, 2002), parfois très complexe pour certaines essences tropicales (Detienne et al., 1998). Sur chaque section, deux à trois rayons ont été identifiés (Photos 7a et 7b) et photographiés et à très haute résolution (1200 dpi). Cette acquisition a été réalisée grâce à une loupe binoculaire Leica (M80) équipée d'une caméra digitale. Les photographies prises sur chaque rayon ont été assemblées avec le logiciel Adobe (R) Photoshop (R) CS2.
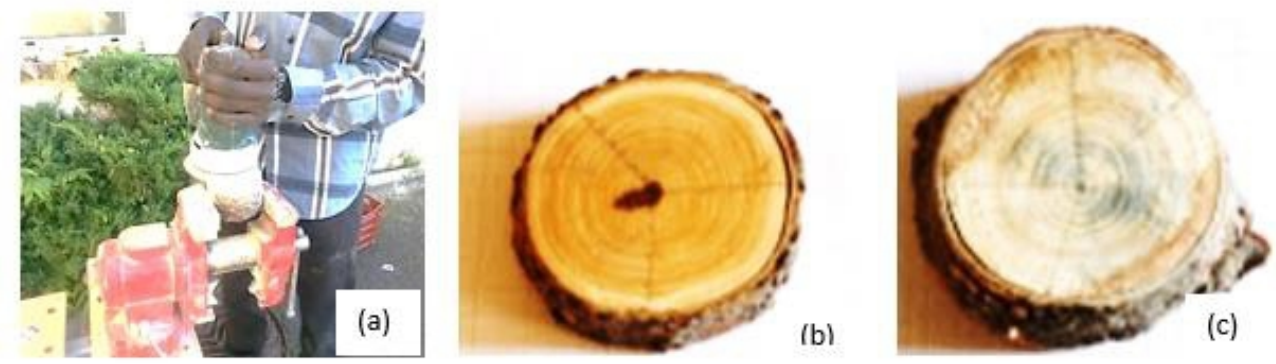

Photo 7. Rondelles : (a) Ponçage ; (b) rondelles de A. raddiana ; (c) rondelles de A. senegal

(Source : Photos prisent lors des travaux de laboratoire en 2015)

\section{Détermination de l'âge des individus}

La détermination de l'âge des individus est un processus qui se déroule en plusieurs étapes. Le long de chaque rayon de pointage, on procède à la mesure de la largeur des cernes au moyen du logiciel Cybis CooRecorder_v7.8 et CDendro_v7.8. Le pointage ou marquage des cernes a été effectué le plus souvent sur deux rayons (R1 et R2) tracés perpendiculairement à la moelle au niveau de chaque rondelle.

Traditionnellement, dans les études dendrochronologiques, les séries élémentaires sont mesurées le long de différents rayons puis interdatées et moyennées afin d'obtenir une série moyenne individuelle. Le logiciel compare aisément les deux séries élémentaires d'un même individu pour s'assurer de leur parfait synchronisme ; synonyme d'un nombre de cernes identique et une 
même année de formation. Cette analyse permet de déterminer l'âge de l'arbre. La qualité de l'interdatation a été évaluée au moyen de l'algorithme de corrélation croisée développée par Baillie et Pilcher (1973). Les valeurs du $t$ de Student ont été calculées pour toutes les superpositions possibles entre deux séries :

$$
t=\frac{|r|}{\sqrt{1-r^{2}}} * \sqrt{(n-2)}
$$

Où $r$ désigne le coefficient de Pearson pour l'échantillon et $\mathrm{n}$ le nombre de cernes de la série.

En complément du t-test, le Gleichläufigkeitkoeffizient (GLK), communément utilisé en dendrochronologie a été calculé. GLK exprime le pourcentage de tendances (augmentation ou diminution de croissance) synchrones de deux séries (Eckstein et Bauch, 1969) :

$$
\begin{array}{ll}
\Delta \mathrm{i}>0 ; G_{i x}=+\frac{1}{2} & \Delta \mathrm{i}=\left(x_{i+1}-x i\right) \\
\text { when } \Delta \mathrm{i}=0 ; G_{i x}=+0 & \Delta \mathrm{i}<0 ; G_{i x}=-\frac{1}{2}
\end{array}
$$

Pour deux courbes x et y : $G_{(x, y)}=\frac{100}{n-1} \sum_{i=1}^{n-1}\left|G_{i x}+G_{i y}\right|$

Où $\mathrm{G}$ représente le Gleichläufigkeit, $\mathrm{n}$ le nombre de valeurs, $\mathrm{x}$ et y les séries temporelles élémentaires ou individuelles analysées et $\Delta \mathrm{i}$ la différence de largeur entre deux cernes consécutifs. Le test t représente le degré d'affinité de deux séries temporelles et intègre le nombre d'observations (Baillie et Pilcher, 1973). GLK est une méthode non-paramétrique pour l'analyse des concordances. La combinaison des deux méthodes permet d'optimiser l'interdatation (Cook et Kairiukstis, 1990). La tendance d'âge associée à l'augmentation du diamètre a été retirée de chaque série individuelle (Cook et Kairiukstis, 1990) grâce à une moyenne mobile unilatérale calculée sur une période de 5 ans (Rinn et Jäkel, 1997). La sensitivité moyenne de la chronologie a été calculée selon la formule ci-dessous :

$S=\frac{\sum_{i=1}^{n-1}\left|S_{i}+1\right|}{n-1}$ avec $S_{i}=\frac{\left(x_{i}-x_{i-1} * 2\right.}{\left(x_{i}+x_{i-1}\right)}$

où $x$ est la largeur de cerne et $n$ le nombre total de cernes d'un individu. La sensitivité moyenne fournit des indications sur la variabilité interannuelle et l'impact des paramètres environnementaux sur la croissance (Schweingruber, 1988).

\section{Résultats}

\section{Structure morphologique des cernes}

La figure 2 présente la structure morphologique des deux espèces étudiées. Elle montre que A. raddiana et $A$. senegal ont des cernes de croissance visibles et nets, minces, clairs et séparés par des bandes de parenchymes. Chez A. raddiana la limite d'accroissement des cernes annuels 
est relativement discrète. Les limites de croissance ont été identifiées avec des parenchymes étroits. Cependant, ces structures sont souvent discontinues à l'intérieur de la section de l'individu. La détection est parfois perturbée du fait : (i) de cernes partiels, discontinus ou extrêmement étroits, particulièrement fréquents sur la partie externe des disques où les cernes de croissance ont tendance à être plus étroits ; (ii) de nombreux rayons ligneux qui interrompent la continuité de cette démarcation (Figure. 2) ; (iii) d'amas de parenchymes de taille pluri-millimétriques qui masquent la fine bande de parenchyme marginal ; (iv) de la présence de bourrelets cicatriciels ; (v) de dommages cambiaux fréquents causés par des insectes xylophages tels que Sinoxylon senegalensis (coléoptère de la famille des Bostrichideae).
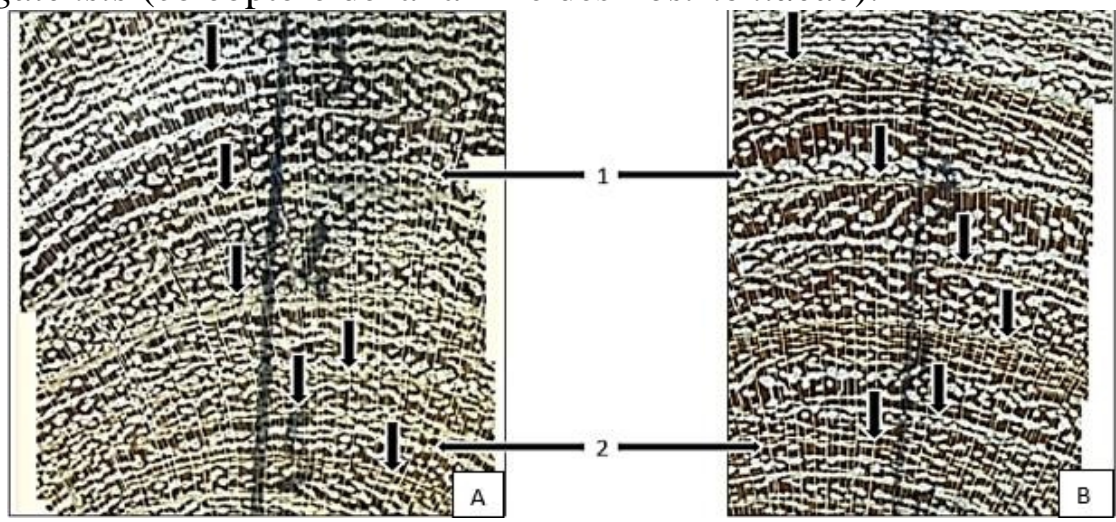

Figure 2 : Structure de cernes de croissance : A. Chez A. raddiana; B. Chez A. senegal (1.Parenchyme; 2. Rayon ligneux)

\section{Critères caractéristiques de l'analyse des cernes}

Les observations découlant de l'analyse de l'anatomie structurale et morphologique des cernes, nous ont permis de présenter quelques critères caractéristiques des cernes chez ces deux espèces (Tableau I).

Tableau I : Quelques critères caractéristiques de l'analyse des cernes

\begin{tabular}{|c|c|c|}
\hline \multirow{2}{*}{ Critères d'observations } & \multicolumn{2}{|c|}{ Espèces } \\
\cline { 2 - 3 } & A. raddiana & A. senegal \\
\hline Bois final de couleur sombre & ++ & ++ \\
\hline Ligne terminale continue ou interrompue & \pm & \pm \\
\hline Possibilité de lecture des cernes & \pm & \pm \\
\hline Cernes nuls ou partiellement nuls & 0 & 0 \\
\hline Présence de faux cernes & \pm & \pm \\
\hline Présence de doubles cernes & ++ & 0 \\
\hline
\end{tabular}

(++ $=$ toujours présent $; \pm=$ plus ou moins présent ou distinct ; $0=$ absent $)$

\section{Intra-datation de cernes et détermination de l'âge des arbres}

Les séries élémentaires mesurées le long des deux ces rayons puis interdatées et moyennées ont permis d'obtenir une série moyenne individuelle ou chronologie individuelle. Un exemple d'intra-datations est présenté chez 
A. raddiana (Figure. 3) et $A$. senegal (Figure. 4). Ces intra-datations nous ont permis, d'une part de vérifier la qualité du marquage des cernes et d'autre part de voir l'allure et la synchronisation entre les cernes de croissance. La qualité des intra-datations dépend de certains paramètres comme le coefficient de corrélation (CorrC) et le Ttest qui montre la relation existant entre coefficients de corrélation et l'âge de l'individu. Selon l'étude de Munaut (1966) une intradatation est le gage d'une bonne détermination de l'âge des individus lorsque le coefficient de corrélation (CorrC) est dans l'intervalle (0.32-0.83) pour des séries de longueurs comparables.

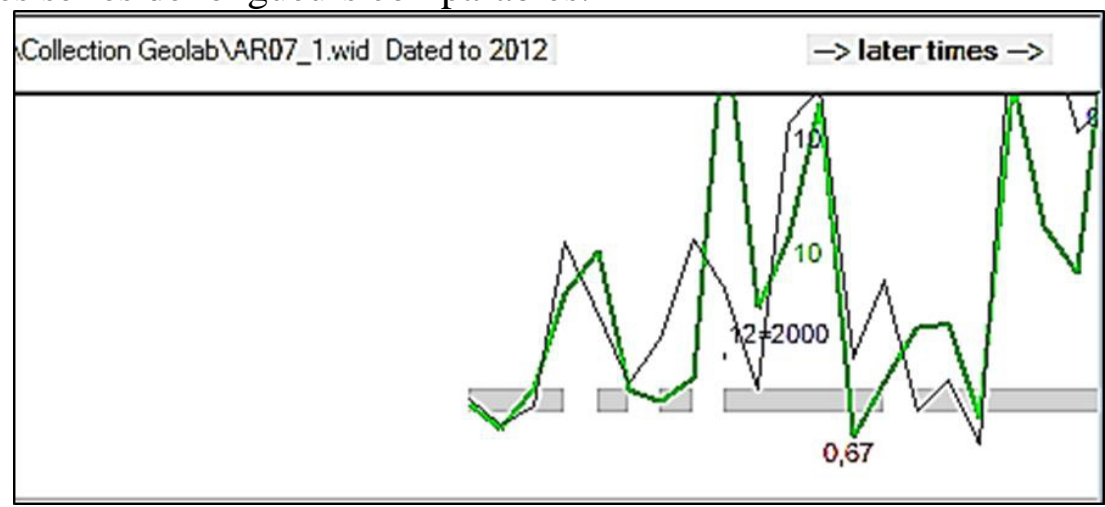

Figure 3 : Exemple d'intradatation des rayons R1 et R2 pour l'individu ACAR7 (A. raddiana). Le coefficient de corrélation est de 0.63 et l'âge estimé de 14 ans. Les deux courbes représentent les rayons $\left(\mathrm{R}_{1}\right.$ et $\left.\mathrm{R}_{2}\right)$ tracés dans chaque rondelle.

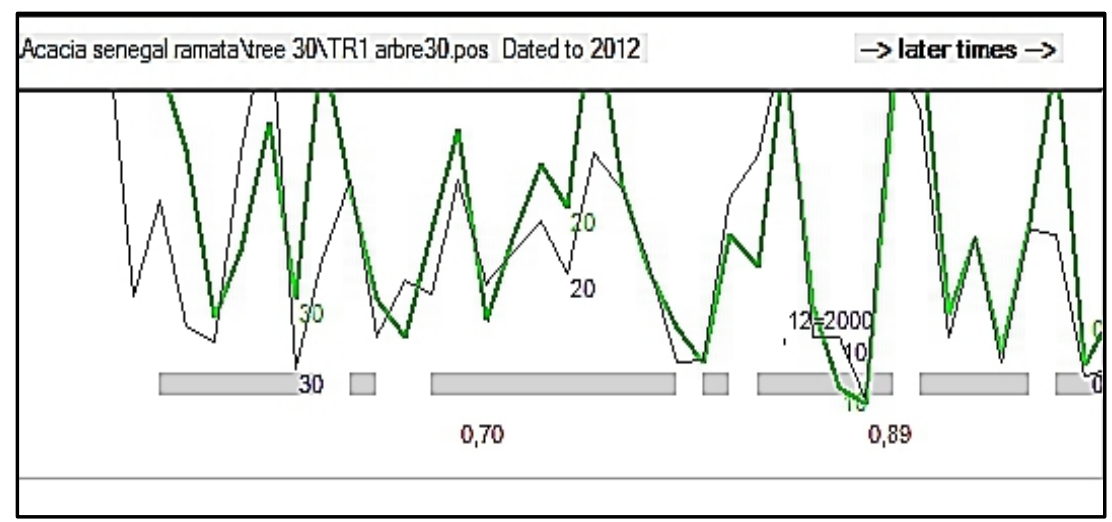

Figure 4 : Intradatation des rayons R1 et R2 de l'individu ACAS30 (A. senegal), $r=0.82$; âge 35 ans.

L'intra-datation des rayons de chaque rondelle a facilité le comptage des cernes et éventuellement l'estimation de l'âge des différents individus de chaque essence. Les résultats sont résumés dans le tableau II. Les forts coefficients de corrélation (CorrC) ainsi que les Ttest montrent la relation existant entre coefficients de corrélation et âges mesurés avec les rayons intraindividuels (R1 et R2). 
Tableau II : Ages des individus chez A. raddiana et A. senegal

\begin{tabular}{|c|c|c|c|c|c|c|c|c|c|}
\hline \multicolumn{5}{|c|}{ Acacia raddianna (ACAR) } & \multicolumn{5}{|c|}{ Acacia senegal (ACAS) } \\
\hline ARBRE & $\begin{array}{c}\text { Rayon } \\
\text { de } \\
\text { pointag } \\
\text { e }\end{array}$ & CorrC & TTest & $\begin{array}{c}\text { AGES } \\
\text { (overlap) }\end{array}$ & ARBRE & $\begin{array}{l}\text { Rayon de } \\
\text { pointage }\end{array}$ & CorrC & TTest & $\begin{array}{c}\text { AGES } \\
\text { (overlap) }\end{array}$ \\
\hline ACAR1 & R1-R2 & 0.91 & 5,1 & 26 & ACAS3 & R1-R2 & 0,71 & 5 & 27 \\
\hline ACAR5 & R1-R2 & 0,75 & 3,4 & 11 & ACAS4 & R1-R2 & 0,50 & 2,3 & 18 \\
\hline ACAR7 & R1-R2 & 0,63 & 2,8 & 14 & ACAS6 & R1-R2 & 0,71 & 3,9 & 17 \\
\hline ACAR10 & R1-R2 & 0,74 & 4,1 & 26 & ACAS8 & R1-R2 & 0.54 & 2,5 & 19 \\
\hline ACAR14 & $\mathrm{R} 1-\mathrm{R} 2$ & 0,78 & 5,2 & 19 & ACAS9 & R1-R2 & 0,67 & 3,5 & 17 \\
\hline ACAR15 & R1-R2 & 0,50 & 2,6 & 21 & ACAS11 & R1-R2 & 0,62 & 7,4 & 22 \\
\hline ACAR16 & R1-R2 & 0,55 & 1,7 & 19 & ACAS12 & R1-R2 & 0,61 & 2,6 & 13 \\
\hline ACAR18 & R1-R2 & 0,56 & 3,4 & 27 & ACAS23 & $R I-R 2$ & 0,84 & 7,4 & 22 \\
\hline ACAR19 & R1-R2 & 0,35 & 1,7 & 17 & ACAS24 & R1-R2 & 0,67 & 5,3 & 30 \\
\hline ACAR20 & $R 1-R 2$ & 0,52 & 2,7 & 22 & ACAS26 & R1-R2 & 0,74 & 4,8 & 21 \\
\hline ACAR24 & $R 1-R 2$ & 0,76 & 5,5 & 24 & ACAS27 & R1-R2 & 0,80 & 6,6 & 35 \\
\hline ACAR25 & $R 1-R 2$ & 0,33 & 1,5 & 21 & ACAS30 & R1-R2 & 0,82 & 8,3 & 35 \\
\hline ACAR28 & $\mathrm{R} 1-\mathrm{R} 2$ & 0,34 & 1,7 & 24 & & & & & \\
\hline ACAR29 & $\mathrm{R} 1-\mathrm{R} 2$ & 0,71 & 2,9 & 10 & & & & & \\
\hline
\end{tabular}

L'analyse du (Tableau. II), nous montrent que chez A. raddiana l'individu cadet (ACAR29) a un âge de 10 ans et le pied le plus âgé (ACAR18) à 27 ans. Cependant, les individus qui ont présenté les meilleurs coefficients de corrélation sont ceux qui présentent souvent les rondelles les plus concentriques avec le moins de défaut de croissance (ACAR5; ACAR10 ; ACAR24 ; ACAR29).

Chez A. senegal les individus sont généralement plus âgés par rapport aux individus de A. raddiana. Les individus les plus âgés (ACAS27 ; ACAS30) ont 35 ans et l'âge minimal est de 13 ans (ACAS12). On observe également une bonne synchronisation intra-individuelle des cernes de croissance des A. senegal avec des coefficients de corrélation comprissent le plus souvent entre 0,61 à 0,84 dont seulement deux individus (ACAS4; ACAS8) présentant des coefficients de corrélation à 0,50 et 0,54 .

\section{Discussion}

Les résultats de cette étude montrent que les Acacias (A. raddiana et A. senegal) présentent des cernes nets, clairs, minces, séparés par des bandes de parenchyme. Les cernes de ces deux espèces présentent également des caractères très semblables. Ceci pourrait s'expliquer par la proximité génétique de ses deux essences. Le phénomène des cernes nuls est très rare dans ces essences. Il est certain que le comptage des cernes est plus ou moins difficile. Cette difficulté serait liée le plus souvent à la discontinuité des cernes accompagnée par une forte densité de bandes de parenchymes très fréquents 
chez A. senegal. Cependant, chez A. raddiana les cernes sont plus concentriques, moins discontinus et la surface transversale du bois est moins perturbée par des anomalies de la croissance ou externe. Des résultats similaires sur la structure des cernes des Acacias ont été présentés par Gammadid (1989), Gourlay (1995a ; 1995b ; 1992) et Steenkamp et al., (2008) qui ont également décrits la présence de ces anneaux. Les limites de croissance ont été identifiées avec des parenchymes étroits. Selon Worbes (1989), les zones de croissance de toutes les légumineuses sont séparées par ces parenchymes en bandes qui masquent le plus souvent le tracé des cernes de croissance. Grace, à des séries d'intra et interdatations de cernes de croissances, les études de Gourlay (1995) et de Eshete et Staêhl, (1999), ont montré le caractère annuel des cernes de croissance chez les Acacias. Les séries d'intradatation de cernes réalisées avec nos échantillons confirment avec de bons coefficients de corrélations (CorrC) (Tableaux II et III), que chaque année les Acacias produisent un cerne de croissance. Un suivi dendrométrique (non-présenté-ici) (Photo 8), mis en place également depuis 2016 sur six individus plantés au niveau de la Grande Muraille Verte sénégalaise - confirme l'existence de croissance asynchrone. Les individus équipés de dendromètres (deux (02) pieds $A$. raddiana, deux (02) pieds de $A$. senegal et de Balanites aegyptiaca) montrent une reprise de croissance s'étalant entre juillet et mi-août 2017 et une entrée en dormance entre octobre et mi-novembre 2017. En revanche, l'absence d'activité cambiale significative pendant les saisons sèches 2016 et 2017 confirme l'existence d'un cycle annuel de croissance en zone sahélienne.

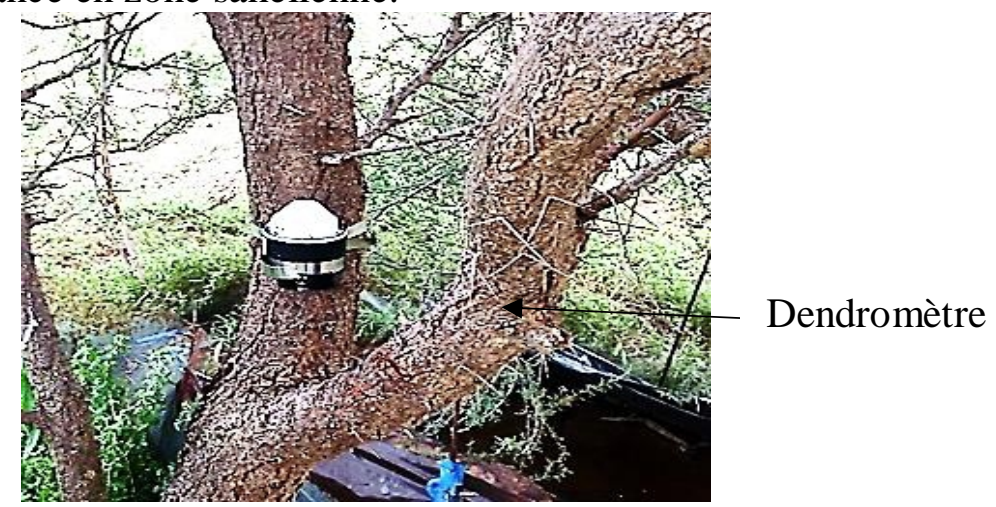

Photo 8: Pied de A. senegal équipé d'un dendromètre

Ainsi, le marquage et le comptage des cernes ont été effectués sur deux rayons pris perpendiculairement par rapport à la moelle. Ils ont donné des corrélations intra-individuelles riches comme chez la pluspart des études d'interconnexion interne des cernes réalisées sur beaucoup d'essences en zone semi-aride ou aride d'Afrique (Gammadid, 1989 Gourlay, 1992; Gebrekirstovet al. (2008) ; Mbow 2009). La présence de cernes discontinus (chez A. raddiana et $A$. senegal) affecte les interdations et handicapent les corrélations entre les 
individus. Ces difficultés sont signalées par Worbes (1995), Tarhule et Hughes (2002). L'impact de l'exploitation anthropique (bois, élagage, blessure des écorces...) fait que certains individus présentent des cernes flous, doubles, discontinus, incomplets qui altère la qualité de l'intra-datation des individus. Selon Eshete et Staêhl (1999), d'autres facteurs, tels que la météo ou la phénologie, la position topographique des arbres échantillonnés sur le terrain (pente, bas-fond, aspect, etc.) peuvent également avoir une influence significative sur la formation des cernes, rendant ainsi le développement d'une chronologie une tâche difficile. Cette asynchronisation des cernes est également signalée par Groenendijet al., (2014) en milieu tropical. Ce qui fait dire à Tarhule et Hughes (2002) que la vitesse de croissance des arbres en zone tropicale sèche, analysée par les cernes, est très variable selon l'espèce botanique, mais aussi selon la station et l'individu. Les études en Afrique de l'Ouest de Tarhule et Hughes (2002) révèlent que la potentialité d'utiliser les cernes pour l'analyse de la croissance des ligneux donne des performances variables selon les espèces. Selon leur étude, il existe une liste d'espèces qui ne permettent pas d'analyser de façon adéquate les cernes des arbres. Dans ce groupe, on note des espèces comme: Lannea acida A. Rich, Lannea microcarpa (ENG), Lespedeza velutina (Bickn), Sclerocarya birrea (A. Rich.) Hochst, Anogeissus leiocarpus (DC.) Guill. \&Perr, Combretum glutinosum auct, Combretum micranthum G. Don., C. nigricans Lepr. ex Guill. \& Perr., Clerodendrum paniculatum L., Terminalia laxiflora Engl., Terminalia macroptera Guill \& Perr., Hymenocardia acida (Delile) A. Chev., Faidherbia albida (Delile) A. Chev, Acacia macrostachya Reichenb.ex DC, Ficus sp., Gardenia sp. et Balanites aegytiaca (L.) Del. Selon (Mbow 2009), les cernes développés en période de sécheresse sont très proches pour être discriminables. La sécheresse peut entraîner aussi des déviations ou fusions de cernes qui s'opèrent de façon irrégulière. Selon (Mariaux 1975 ; Detienne 1989; Worbes 1995), les processus génétiques et les mécanismes d'adaptations développés par certains individus peuvent également affecter la qualité des datations. Cependant, dans notre étude, plusieurs précautions ont été prises afin d'améliorer l'intradatation et de garantir sa précision. Le protocole utilisé et notamment le prélèvement de sections complètes et une sélection minutieuse des échantillons ont permis de s'affranchir partiellement des difficultés évoquées dans la détection des cernes. Ceci nous a permis de bien définir la structure anatomique des cernes de croissance de ces essences et de montrer leur potentialité dendrochronologique. Des corrélations statistiquement significatives comprises entre 0.33 à 0.82 ont ainsi été calculées entre les rayons d'un même individu. Ces valeurs sont comparables à celles obtenues par Munaut (1966) (0.32-0.83) pour des séries de longueurs comparables et les âges déterminés sont aux alentours de 10 à 35 ans chez ces deux Acacia. 


\section{Conclusion}

A partir de l'observation de la structure anatomique des cernes et de la détermination de l'âge des individus par espèce, il en ressort que A. raddiana et $A$. senegal présentent des cernes clairs, minces, séparés par des bandes de parenchyme et des nombreux rayons ligneux qui sont des lames mortes dans le duramen et vivants dans l'aubier. La lecture des cernes est plus facile chez A. raddiana, car présentant les cernes les plus concentriques avec moins de défauts de croissance. Par compte, elle est plus délicate et complexe chez $A$. senegal caractérisée par des cernes doubles, discontinus, interrompus, qui altèrent souvent le marquage des cernes de croissance. La datation des cernes de chaque individu a été effectuée d'une part avec le pointage ou marquage des cernes, et d'autre part par une corrélation intra individuel permettant de montrer la qualité des corrélations et de compter les cernes afin de déterminer l'âge des individus de chaque espèce. Ainsi, cette étude est une ouverture pour mieux élargir la connaissance de la dendrochronologie d'espèces sahéliennes. Elle permet de comprendre l'utilisation des cernes de croissance comme un moyen de datation, mais vise particulièrement à mieux approfondir la connaissance sur la formation et la structure des cernes par: (i) l'analyse de l'anatomie des cernes de croissance ; (ii) la mise en évidence de leur caractère saisonnier par inter-datation des séries annuelles de largeurs de cernes de plusieurs individus; (iii) l'identification des facteurs climatiques expliquant la croissance radiale des espèces sahéliennes. Il sera surtout d'une importance capitale de mettre en place des stratégies et des méthodes permettant de mieux matriser leur vitesse de croissance.

\section{References :}

1. Baillie, M.G.L., and J. R. Pilcher. (1973). A simple cross dating program for tree-ring research. Tree-Ring Bull. 33, pp. 7-14.

2. Bräker O.U. (2002). Measuring and data processing in tree-ring research - a methodological introduction. Dendrochronologia, 20/12, pp. 203-216.

3. Cristopher P. (1991). Acacia tortilis:Fodder tree for deserf sands, $\mathrm{N}$ T A, pp. 91-01.

4. Cook E.R., Kairiukstis L.A.E., 1990. Methods of dendrochronology: Applications in the environmental sciences. - Kluwer Academic Publishers, 408 pages.

5. Coster CH., (1927). Zur anatomie und physiologie der zuwachszonenund jahresringbildung in der tropen Ann. Jard. Bot. Buitenz., 37, pp. 49-161.

6. Coster CH., (1928). Zur anatomie und physiologie der zuwachszonenund jahresringbildung in der tropen Ann. Jard. Bot. Buitenz., 38, pp. 1-114. 
7. Dagnelie P., (1998). Statistiques théoriques et appliquées. Brussels: De Boeck, 517 p.

8. Detienne P., Oyono F., Durrieu de Madron L., Robert NASI. (1998). L'analyse des cernes : Application aux études de croissance de quelques essences en peuplements naturels de forêt dense africaine. Projet Forafri, document $\mathrm{n}^{\circ} 15,40 \mathrm{p}$.

9. Detienne, P. (1989). Appearance of periodicity of growth rings in some tropical woods. IAWA Bull. n.s. 10, pp. 123-132.

10. Dezzeo N., Worbes M., Ishii I., \&Herrera R. (2003). Annual tree rings revealed by radiocarbon dating in seasonally flooded forest of the Mapire River, a tributary of the lower Orinoco River, Venezuela. Vegetation 168(1): pp. 165-175.

11. Diallo A. (2011). Caractérisation de la végétation et des sols dans les plantations d'Acacia senegal (L.) Willd dans la zone de Dahra - sud Ferlo sableux. Thèse de doctorat, UCAD-FST, Département Biologie Végétale, $127 \mathrm{p}$.

12. Diallo A., Faye M. N. et Guissé A., 2011. Structure des peuplements ligneux dans les plantations d'Acacia 415-427.

13. Dione M., (1996). Recherches expérimentales sur le gommier Acacia senegal dans le Ferlo sénégalais, thèse, 135p.

14. Diop A. T. (2007). Dynamique écologique et évolution des pratiques dans la zone sylvo-pastorale du Sénégal: perspectives pour un développement durable. Dakar: Université Cheikh Anta Diop, thèse de doctorat, $387 \mathrm{p}$.

15. Diouf M., (2003). Caractéristiques fondamentales de la feuillaison d'une espèce ligneuse sahélienne : Acacia tortilis (forsk) hyane, variation selon les microsites topographiques du Ferlo (nord-Sénégal). Thèse de doctorat de $3^{\text {ém }}$ cycle FST-UCAD. $103 \mathrm{p}$.

16. Eckstein, D., Bauch, J., (1969). Beitrag zur Rationalisierung eines dendrochronologischen Verfahrens und zur Analyse seiner Aussagesicherheit. Forstwissenschaftliches Central- blatt 88, pp. 230 250.

17. Eshete, G., Ståhl, G. (1998). Functions for multi-phase assessment of biomass in acacia woodlands of the Rift Valley of Ethiopia. For. Ecol. Manage. 105, pp. 79-90.

18. Fichtler E., Clark A. D. \&Worbes M. (2006). Age and Long-term Growth of Trees in an Old-growth Tropical Rain Forest, Based on Analyses of Tree Rings and ${ }^{14}$ C. Biotropica, 35(3): pp. $306-317$.

19. Gammadid, I.D. (1989). Study of growth rates of Acacia bussei (Harms ex.) Sjostedt in the bay region of Somalia. Commonw. For. Rev. 68, pp. 27-35. 
20. Gebrekirstos, A., Mitlohner, R., Teketay, D., Worbes, M., (2008). Climate-growth relationships of the dominant tree species from semiarid savanna woodland in Ethiopia. Trees-Struct Funct 22, pp. 631641.

21. Gourlay I.D. (1992). Age determination of acacias. In: Eggertsson O. (Ed.). Tree Rings and Environment. Proceedings of the international dendrological symposium. Ystad (Sweden), Sept. 3-9, 1990, Lund Univ., 1992, pp. 118-122.

22. Gourlay I.D. (1995a). Growth ring characteristics of some African Acacia species. Journal of Tropical Ecology 11, pp. 121-135.

23. Gourlay I.D. (1995b). The definition of seasonal growth zones in some African Acacia species \pm A review. IAWA Journal 16, pp. 353-359.

24. Guerin. H et al. (1991). Alimentation des ruminants domestiques sur pâturages naturels saheliens et sahelo-soudaniens : étude méthodologique dans la région du Ferlo au Sénégal, Maisons-Alfort, Études et synthèses de l'Institut d'Élevage et de Médecine Vétérinaire des Pays Tropicaux, 1991, 115 p. (ISBN 2859851771).

25. Henry M, Besnard A, Asante WA et al. (2010). Wood density, phytomass variations within and among trees, and allometric equations in a tropical rainforest of Africa. Forest Ecology and Management, 260, pp. 1375-1388.

26. Laporte N., Baccini A., Goezt S., Mekui P., Bausch A. (2010). La première estimation de la biomasse ligneuse aérienne d'Afrique sur la base d'images satellites et inventaires forestiers. Proceeding of the conference carbone stock fluxes, COMIFAC, Brazzaville, March 2010 pp. 58-65.

27. Leprun JC. (1971). Nouvelles observations sur les formations dunaires sableuses fixées du Ferlo nord occidental (Sénégal). Et. Quaternaires ouest africaines. Bull.Liaison, Sénégal., 31: pp. 69-78.

28. Mariaux A. (1967) : Les cernes dans les bois tropicaux africains. Nature et périodicité. Bois et Forêts des Tropiques 113: 3-14 \& 114, pp. 23-37.

29. Mariaux A. (1969). La périodicité de formation des cernes dans le bois de Limba. Revue Bois et Forêts des Tropiques 128, pp. 39-54

30. Mariaux A. (1970). La périodicité de formation des cernes dans le bois de l'Okoumé. Bois et Forêt des Tropiques 131, pp. 37-50.

31. Mariaux A. (1975). Essai de dendrochronologie en climat sahélien sur Acacia raddiana, Bois et Forêts des tropiques, 163, pp 25- 35.

32. Munaut A.V., 1966. Recherches dendrochronologiques sur Pinus sylvestris. 1. Etude de 45 pins sylvestres récents originaires de Belgique. - Agricultura, 14/2, pp. 193-232. 
33. Miehe S., Kluge J., Wehrden H., Retzer V. (2010).Long-term degradation of Sahelian rangeland detectedby 27 years of field study in Senegal Journal compilation 2010 British Ecological Society, Journal of Applied Ecology, 47, pp. 692-700.

34. Mbow C. (2009). Potentiel et dynamique des stocks de carbone des savanes soudaniennes et soudano-guinéennes du Sénégal. Thèse de doctorat d'Etat ès sciences UCAD, $319 \mathrm{p}$.

35. Ndiaye O., 2014. Caractéristiques des sols, de la flore et de la végétation du Ferlo, Sénégal. Thèse de doctorat unique en biologie végétale, option écologie, FST-UCAD, 114p.

36. Ndiaye O., Diallo A., Sagna M. B., et Guisse A. (2013). « Diversité floristique des peuplements ligneux du Ferlo, Sénégal », VertigO - la revue électronique en sciences de l'environnement [En ligne], Volume 13 Numéro 3 décembre 2013, mis en ligne le 24 janvier 2014, consulté le 03 février 2014, pp. 12. URL : http://vertigo.revues.org/14352; DOI : 10.4000/vertigo. 14352

37. Niang K. (2009). L'arbre dans les parcours communautaires du FerloNord (Sénégal). Mémoire de DEA en biologie végétale, FST-UCAD, $67 \mathrm{p}$.

38. Ngom D., Charahabil, M M., Sarr, O., Bakhoum A., et. Akpo L E. (2014). Perceptions communautaires sur les services écosystémiques d'approvisionnement fournis par le peuplement ligneux de la Réserve de Biosphère du Ferlo (Sénégal). VertigO - la revue électronique en sciences de l'environnement, Volume 14 Numéro 2 (septembre 2014), $18 \mathrm{p}$.

39. Patrut A., VON Reden K. F., Lowy D. A., Alberts A. H., Pohlman J. W., Wittmann R., Gerlach D., XU L. \&Mitchell C. S. (2007). Radiocarbon dating of a very large African baobab. Tree Physiology 27: pp. 1569-1574.

40. Peiry L P., Voldoire O. (In press). Cadre Climatique et ressouce en eau dans la zone de la Grande Muraille Verte au Sénégal (GMVS). In Boestch. G et Duboiz P (Edition) la Grande Muraille Verte en marcheCNRS, Edition-Paris 15p.

41. Poupon H., 1979. Structure et dynamique de la strate ligneuse d'une steppe sahélienne au nord du Sénégal. Thèse Sciences Naturelles, Université de Paris Sud, ORSAY. Travaux et Document de l'ORSTOM, 351p.

42. Sarr M. A. (2009). Évolution récente du climat et de la végétation au Sénégal (cas du Ferlo), Thèse de doctorat, Université Jean Moulin Lyon 3, LCRE UMR 5600 CNRS, 410 p. 
43. Sarr O., Bakhoum A, Diatta S., Akpo L E. (2013). L'arbre en milieu soudano-sahélien dans le bassin arachidier (Centre-Sénégal). Journal of Applied Biosciences 61, pp. 4515 - 4529.

44. Steenkamp C.J., Vogel J.C.,Fuls A., VanRooyenN., M.W. (2008). Age determination of Acacia erioloba trees in the Kalahari. Journal of Arid Environments, 72/44, pp. 302-313.

45. Stokes, M.A., Smiley, T.L., (1996). An Introduction to Tree-Ring Dating. The University of Arizona Press, Tuscon, $73 \mathrm{pp}$.

46. Tarhule, A., Hughes M. (2002). Tree-ring research in semi-arid West Africa: need and potential. Tree-ring Research, 58 (1/2), pp. 31-46.

47. Vassal J.(1993). Un exemple de biodiversité : le genre Acacia subgen.heterophyllum. Acte de colloque international de Phytogéographie tropicale. Paris, pp. 365-377.

48. Von Maydell, H. J. (1990). Arbres et arbustes du Sahel: leurs caractéristiques et leurs utilisations. GTZ, Verlag Josef Margraf, Eschborn, 531p.

49. Worbes M. (1989). Growth rings, increment and age of trees in inundation forests, savannas and a mountain forest in the Neotropics. IAWA Bull. n.s. 10, pp. 109-122.

50. Worbes M. (1995). How to measure growth dynamics in tropical trees. A review. IAWA Journal, 16 (4), pp. 337-351. 